\title{
Sensing Properties of Femtosecond Laser-Inscribed Long Period Gratings in Photonic Crystal Fiber
}

\author{
Kyriacos KALLI ${ }^{1}$, Tom ALLSOP ${ }^{2}$, Kaimin ZHOU $^{2}$, Graham SMITH ${ }^{2}$, \\ Michael KOMODROMOS ${ }^{3}$, David $\mathrm{WEBB}^{2}$, and Ian BENNION ${ }^{2}$ \\ ${ }^{1}$ Nanophotonics Research Laboratory, Department of Electrical Engineering and Information Technology, Cyprus \\ University of Technology, Limassol, 3036 Cyprus \\ ${ }^{2}$ Photonics Research Group, Aston University, Birmingham, B4 7ET, United Kingdom \\ ${ }^{3}$ Department of Electrical Engineering, Frederick University, Filokyprou 7, Nicosia, Cyprus \\ *Corresponding author: Kyriacos KALLIＥ-mail: Kyriacos.Kalli@cut.ac.cy
}

\begin{abstract}
The use of near infrared, high intensity femtosecond laser pulses for the inscription of long period fiber gratings in photonic crystal fiber is reported. The formation of grating structures in photonic crystal fiber is complicated by the fiber structure that allows wave-guidance but that impairs and scatters the femtosecond inscription beam. The effects of symmetric and asymmetric femtosecond laser inscriptions are compared and the polarization characteristics of long period gratings and their responses to external perturbations are reported.
\end{abstract}

Keywords: Fiber gratings, femtosecond laser inscription, photonic crystal fiber

\section{Introduction}

Long period gratings (LPG) result from periodic refractive index variations inscribed axially along the core of an optical fiber and consequently guided light is coupled out of core region, where it interacts with a potentially infinite number of cladding modes. Due to phase matching that occurs between the core and cladding modes, this interaction occurs between discrete modes, producing a characteristic loss spectrum that also occurs at discrete wavelengths [1-6]. The index modulation change within the core of a single mode optical fiber can vary depending upon the inscription method used, tending to be in the range of $10^{-4}$ to 0.1 , whereas the period for LPGs is typically between $100 \mu \mathrm{m}$ and $600 \mu \mathrm{m}$. The inscription can be induced by using ultra-violet laser irradiation (using photosensitive single mode optical fiber) [1]; through fusion arc or laser heating inscription (for non-photosensitive optical fiber $[7,8]$, with an inscription mechanism dominated by compaction and structural change) or more recently by femtosecond lasers [9-11], where in the near infrared (from $800 \mathrm{~nm}$ to $1100 \mathrm{~nm}$ ) the dominant inscription mechanism appears to be a combination of effects that include void creation, material compaction, and the photo-elastic effect induced by thermal strain. There is significant interest in the use of high intensity femtosecond laser pulses for inscribing fiber gratings, where the principal advantage is the potential for grating inscription in any fiber type without preprocessing or special core doping.

The formation of grating structures in photonic crystal fiber (PCF) has proven difficult, as the holey fiber structures that allow wave-guidance to impair

Received: 23 September 2010 / Revised version: 18 October 2010

C The Author(s) 2011.This article is published with open access at Springerlink.com 
and scatter the femtosecond inscription beam. Therefore the consistent manufacture of long period gratings in photonic crystal fiber is the subject of considerable research interest due to their potential applications as filters and sensing devices, responsive to strain, temperature, bending and refractive index. Compared to fiber Bragg grating (FBG) sensors, LPGs have more complex spectra, usually with broader spectral features.

On the other hand they are intrinsically sensitive to bending and refractive index. Perhaps more importantly, the design of fiber and the choice of grating period can have a considerable influence on the sensitivity of various parameters, for example allowing the creation of a bend sensor with minimal temperature cross-sensitivity. This is not readily possible with FBG sensors. Here we compare the effects of symmetric and asymmetric femtosecond laser inscriptions on LPG wavelength spectra and report on the characterization of the gratings to external perturbations.

\section{Fabrication of grating devices}

A series of LPGs with the same period, $400 \mu \mathrm{m}$, were fabricated in endlessly single mode (ESM) fiber from Crystal Fiber A/S. Several devices were made with approximately the same inscription pulse energy of $(450 \pm 20) \mathrm{nJ}$; a few other devices were written with energy ranging from $410 \mathrm{~nJ}$ to $650 \mathrm{~nJ}$ that required various grating lengths to obtain maximum strength attenuation bands.

The refractive index changes were induced by using a femtosecond laser producing a $1 \mathrm{kHz}$ train of $150 \mathrm{fs}$ pulses at $800 \mathrm{~nm}$.

The femtosecond laser radiation was focused on a predetermined point near the fiber core with a $\times 100(N A=0.55)$ microscope objective, whilst the fiber was translated in a direction parallel to the fiber axis. The translation speed was $100 \mu \mathrm{m} / \mathrm{s}$. A cylindrical lens $(f=100 \mathrm{~mm})$ was placed in close proximity to the microscope objective to circularize the laser beam at the point of inscription, as shown in Fig. 1; other details of the experimental setup can be found elsewhere [9]. As a result of previous investigations into the spectral characteristics of femtosecond laser inscribed LPGs in conventional single mode fibers [12], it was anticipated that devices would be strongly polarization dependent and so the light used to monitor the grating growth during fabrication was polarized, as shown in Fig. 1(b). The polarization of the light was adjusted to maximize the polarization dependent LPG attenuation bands. For the inscription of symmetric LPGs the laser focal point was centered on the fiber core, whereas the focal inscription point was moved off-axis using the translation stage to inscribe asymmetric LPGs.

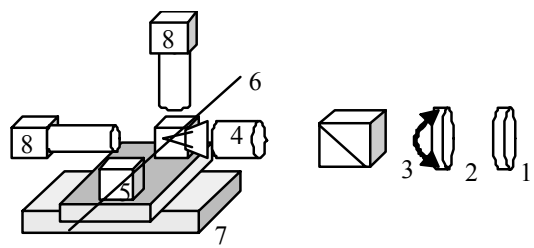

(a)

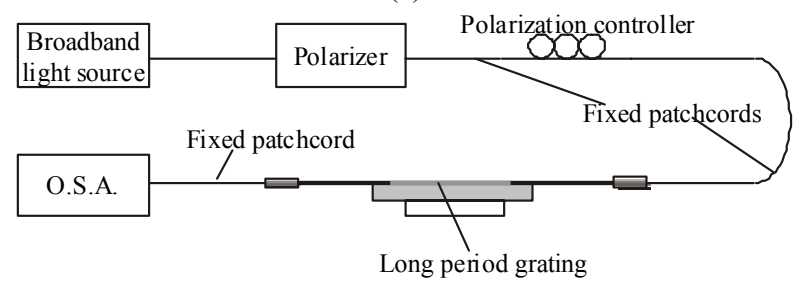

(b)

Fig. 1 (a) Optical layout of femtosecond inscription scheme(1-shutter; 2-half-wave plate; 3-Glan prism; 4$\times 100$ microscopic objective; 5-two alignment 3D translation stages; 6-fiber core; 7-high precision computer controlled 2D stages; 8-CCD-cameras) and (b) schematic of the system monitoring LPG transmission spectra during fabrication.

\section{Laser inscription images}

After inscription, the LPGs were examined with differential interference contrast microscope and the modified regions of the fiber were identified, as shown in Fig. 2. The consistent placement of the inscription laser beam was achieved by precise control of the translation stage system, thus ensuring that LPGs with the same transmission spectra were inscribed. 


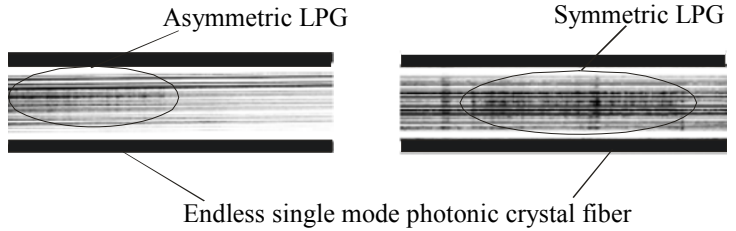

Fig. 2 Images of inscribed sections of photonic crystal fiber, showing asymmetric and symmetric (centered on the core) inscriptions.

\section{Transmission spectra and polarization dependence}

Typical examples of femtosecond laser inscribed LPG transmission spectra are shown in Fig. 3 (one where the structural modification of PCF appears to be radially symmetric to the fiber axis and the other where the structural modification is asymmetric, being displaced from the fiber axis). The plots show the variation of the spectra obtained by adjusting the polarization of illuminating light to obtain the strongest coupling response to each of the attenuation bands presented in the spectrum.

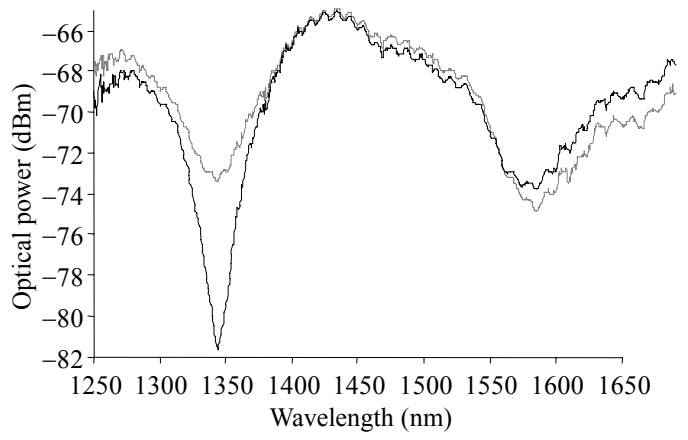

(a)

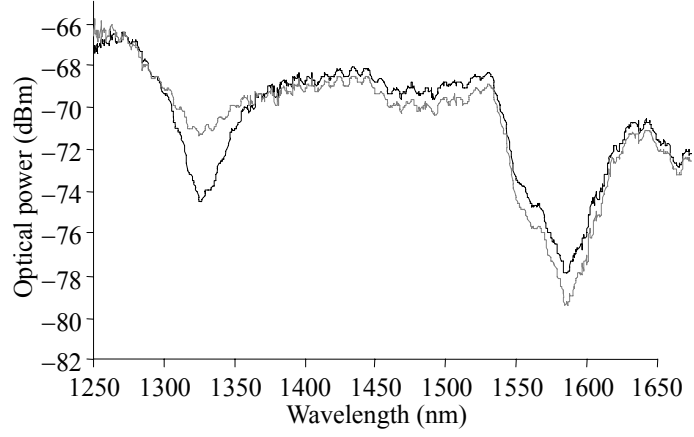

(b)

Fig. 3 Typical transmission spectra of femtosecond laser inscribed LPGs (period: $400 \mu \mathrm{m}$ ) in PCF: (a) asymmetrical inscription (laser energy: 410nJ) and (b) symmetrical inscription (laser energy: $470 \mathrm{~nJ}$ ), highlighting the variation in response to input polarization.
The variations in resonant wavelength between two polarization states for the attenuation band close to $1590 \mathrm{~nm}$ were $1.4 \mathrm{~nm}$ for symmetrically written LPG and $6.4 \mathrm{~nm}$ for asymmetric one with figures of $0.5 \mathrm{~nm}$ and $4.1 \mathrm{~nm}$, respectively, for stop bands at $1350 \mathrm{~nm}$.

The polarization dependence appears to have two main contributions: one is related to the symmetry of inscription and the other is related to the power of inscription. The birefringence varies from $10.2 \mathrm{~nm}$ to $1.4 \mathrm{~nm}$ over the laser inscription energy range used, as shown in Fig. 4.

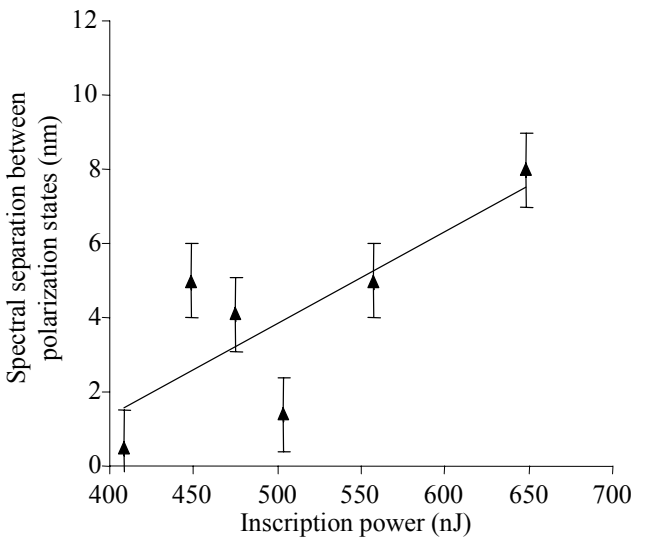

(a)

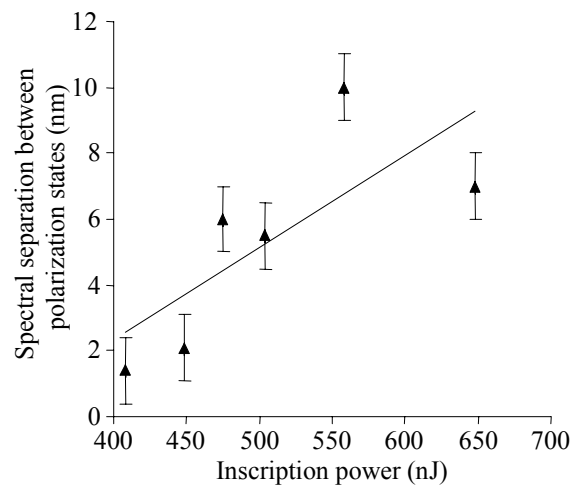

(b)

Fig. 4 Observed maximum wavelength separation of key attenuation bands due to polarization, as a function of laser inscription energy: (a) $1600 \mathrm{~nm}$ and (b) $1350 \mathrm{~nm}$. The lines are linear regressions showing the increasing trend in birefringence with laser inscription energy.

\section{Curvature sensitivity}

To examine the curvature sensitivity of LPGs, the devices were exposed to controlled bending, without fiber twist, by using the rig shown in Fig. 5. 
For asymmetric LPGs, the inscribed perturbation was oriented below the fiber axis in the plane of induced bending. We described the bending as convex bending (a positive curvature) when the fiber was bent upwards and concave bending (a negative curvature) when the fiber was bent downwards (Fig. 5). The bend sensitivity of these LPGs were investigated with a broadband light source and a polarizer, which in turn was connected to a polarization controller; the light from this arrangement illuminated the LPGs and observations of transmitted spectra were made using an optical spectrum analyzer. The polarization controller was used to maximize coupling of illuminating light into one of attenuation bands present in the transmission spectrum of the LPG, and each attenuation band could be studied at a time.

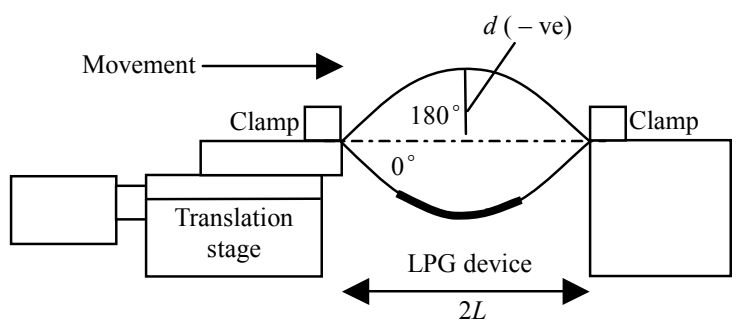

Fig. 5 Curvature test rig used to bend the fiber, giving it positive (convex) curvature and negative (concave) curvature.

The symmetrically inscribed LPGs exhibit no dependence on bend sensitivity with fiber orientation, while asymmetrically inscribed LPGs

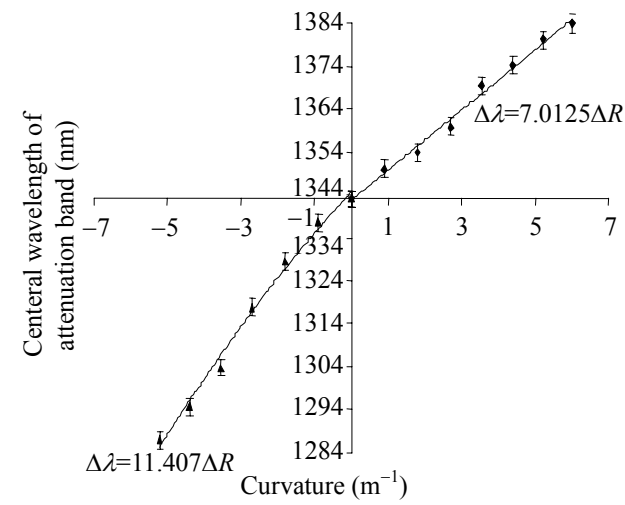

(a) display directional bend sensitivity to some attenuation bands. The transmission spectra of such a LPG (that used for Fig. 3(a)) are shown in Fig. 6.

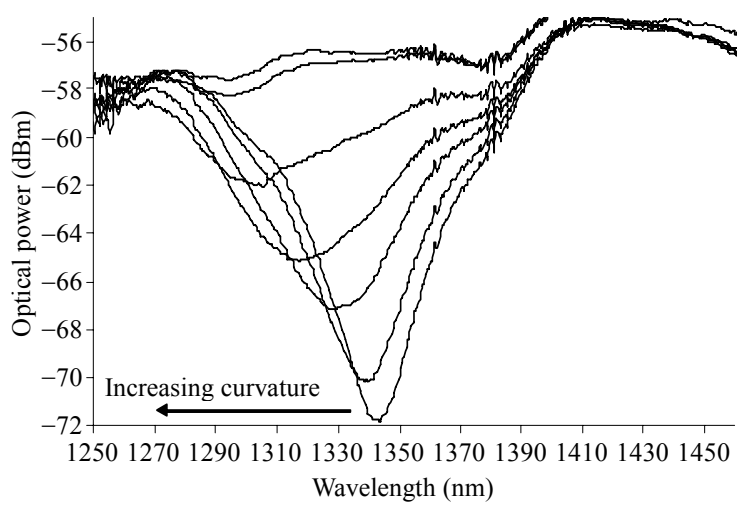

(a)

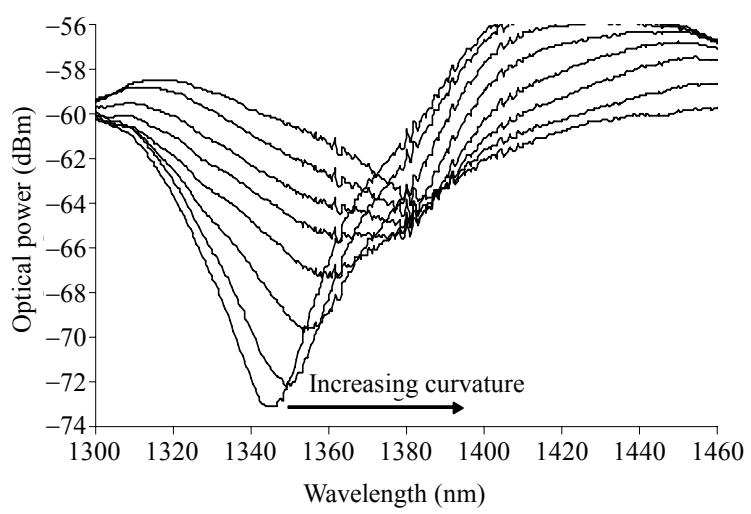

(b)

Fig. 6 An example of the curvature response of transmission spectra for an asymmetrically inscribed LPG written in PCF (period $=400 \mu \mathrm{m}$, length $=96 \mathrm{~mm}$, inscription power $=410 \mathrm{~nJ})$ LPG was subjected to (a) concave and (b) convex bending.

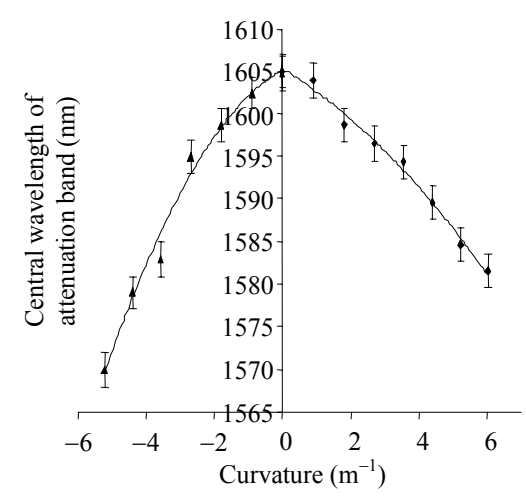

(b)

Fig. 7 (a) and (b) Spectral sensitivity to curvature of both observed attenuation bands for an asymmetrically inscribed LPG in PCF (period $=400 \mu \mathrm{m}$, inscription energy $=410 \mathrm{~nJ}$, length $=9.6 \mathrm{~mm}$ ) for both convex ending and concave bending. 
Spectral sensitivity of two attenuation bands are shown in Fig. 7. The asymmetrically inscribed LPGs in PCF generate some attenuation bands which display a clear directional sensitivity (Fig. 7(a)), whilst others are much closer to giving a symmetrical response (Fig. 7(b)). The E-field profiles of the modes in this PCF have been investigated [13] and show dramatic variations in the radial distribution of the E-field with mode order, implying that we can expect radically different behavior from the various modes.

\section{Temperature sensitivity}

The temperature sensitivity was also investigated. The LPGs were placed on an insulated Peltier cooler and the temperature induced wavelength shift of the attenuation bands was recorded. Temperature sensitivity of $5.7 \times 10^{-3} \mathrm{~nm} /{ }^{\circ} \mathrm{C}$ for the attenuation band at $1350 \mathrm{~nm}$ and $4.2 \times 10^{-3} \mathrm{~nm} /{ }^{\circ} \mathrm{C}$ for the attenuation band at $1600 \mathrm{~nm}$ which are lower values compared to those reported for LPGs in SMF-28 fiber [12] and are in approximate agreement with the results of other studies on PCF based LPGs [7, 8], as shown in Fig. 8.

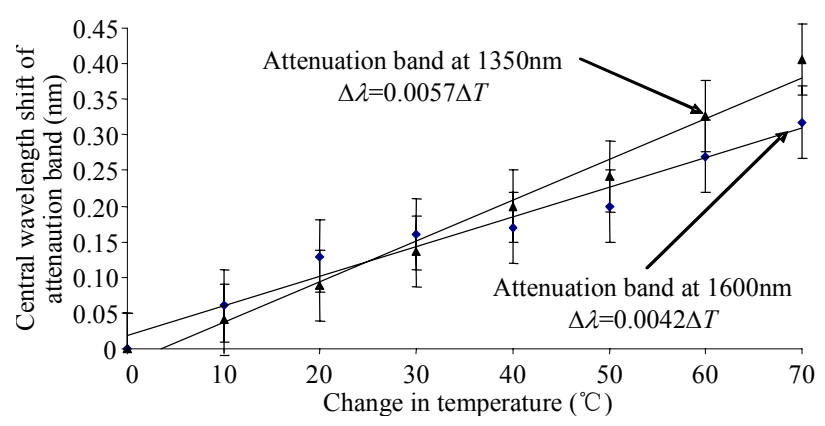

Fig. 8 Spectral sensitivity to temperature for two attenuation bands of the asymmetrically inscribed LPG in PCF (period = $400 \mu \mathrm{m}$, inscription energy $=410 \mathrm{~nJ}$, length $=9.6 \mathrm{~mm}$ ).

\section{Conclusions}

A series of symmetric and asymmetric LPGs were inscribed in PCF using a femtosecond laser system. The asymmetric LPGs were found to be spectrally sensitive to bend orientation with particular attenuation bands yielding a spectral curvature sensitivity of $-11.4 \mathrm{~nm} \cdot \mathrm{m}$ for a concave bend and $7.0 \mathrm{~nm} \bullet \mathrm{m}$ for a convex bend, with low temperature sensitivity. The great advantage with this PCF is the low temperature cross-sensitivity, however, there is an issue with the polarization dependence of the attenuation bands. All LPGs exhibit spectral birefringence ranging from $1 \mathrm{~nm}$ to $10 \mathrm{~nm}$, which appears to be related to the inscription method and the laser energy used to fabricate the LPG. This suggests that these devices have the potential to be used as curvature sensors with high resolution.

\section{Acknowledgement}

This work was supported by the Research Promotion Foundation (Cyprus) under the grant "FEMTO".

Open Access This article is distributed under the terms of the Creative Commons Attribution License which permits any use, distribution, and reproduction in any medium, provided the original author(s) and source are credited.

\section{References}

[1] A. Vengsarkar, P. J. Lemaire, J. B. Judkins, V. Bhatia, T. Erdogan, and J. E. Sipe, "Long period gratings as band-rejection filters," J. Lightwave Tech., vol. 14, no. 1 , pp. 58-64, 1996.

[2] V. Bhatia, "Applications of long-period gratings to single and multi-parameter sensing," Opt. Exp., vol. 4, no. 11, pp. 457-466, 1999.

[3] T. Erdogan, "Fiber grating spectra," J. Lightwave Tech., vol. 15, no. 8, pp. 1277-1294, 1997.

[4] X. Shu, L. Zhang, and I. Bennion, "Sensitivity characteristics of long-period fiber gratings," J. Lightwave Tech., vol. 20, no. 2, pp. 255-266, 2002.

[5] H. Patrick, A. Kersey, and F. Bucholtz, "Analysis of the response of long period fiber gratings to external index of refraction," J. Lightwave Tech., vol. 16, no. 9, pp. 1606-1612, 1998.

[6] T. Erdogan, "Cladding-mode resonances in short- and long-period fiber grating filters," J. Opt. Soc. Am. A, 
vol. 14, no. 8, pp. 1760-1773, 1997.

[7] H. Dobb, K. Kalli, and D. J. Webb, "Temperature insensitive long period grating sensors in photonic crystal fiber," Elec. Lett., vol. 40, no. 11, pp. 657-658, 2004.

[8] H. Dobb, K. Kalli, and D. J. Webb, "Measured sensitivity of arc-induced long-period grating sensors in photonic crystal fiber," Opt. Comm., vol. 260, no. 1, pp. 184-191, 2006.

[9] A. Martinez, M. Dubov, I. Khrushchev, and I. Bennion, "Point by point FBG inscription by a focused NIR femtosecond laser," presented at OSA Conf. Proc. CLEO/IQEC and PhAST, Washington, 2004.

[10] Y. Kondo, K. Nouchi, T. Mitsuyu, M. Watanabe, P. G. Kazans1ky, and K. Hirao, "Fabrication of long-period fiber gratings by focused irradiation of infrared femtosecond laser pulse," Opt. Lett., vol. 24, no. 10, pp. 646-648, 1999.

[11] A. A. Fotiadi, G. Brambilla, T. Ernst, S. A. Slattery, and D. N. Nikogosyan, "TPA-induced long-period gratings in a photonic crystal fiber: inscription and temperature sensing properties," J. Opt. Soc. Am. B, vol. 24, no. 7, pp. 1475-1481, 2007.

[12]T. Allsop, H. Dobb, A. Main, A. Martinez, M. Dubov, K. Kalli, D. J. Webb, and I. Bennion, "Comparison of the spectral properties of high temperature annealed long period gratings inscribed by fs laser, UV, and fusion arc," Proc. SPIE, vol. 6193, pp. 61930M, 2006.

[13] J. Petrovic H. Dobb, V. Mezentsev, K. Kalli, D. J. Webb, and I. Bennion, "Sensitivity of LPGs in PCFs fabricated by an electric arc to temperature, strain, and external refractive index," J. Lightwave Tech., vol. 25, no. 5, pp. 1306-1312, 2007. 